\title{
THE ELEMENTARY CASES IN TEICHMÜLLER'S MAPPING THEOREM
}

\author{
Kurt Strebel
}

\section{Introduction}

1.1. Teichmüller's mapping theorem. Let $R$ be a compact bordered Riemann surface with finitely many distinguished points $P_{\nu}$ in the interior or on the boundary of $R$. The surface $R$ punctured at the $P_{\nu}$ is denoted by $\dot{R}=$ $R \backslash\left\{P_{\nu}\right\}$. The punctures $P_{\nu}$ are called the vertices of $R$, the boundary intervals between the vertices its sides. A quadratic differential $\varphi$ is said to belong to $R$ or to be associated with $R$, if it has the following properties:

(1) $\varphi$ is holomorphic on $\dot{R}$,

(2) $\varphi$ has finite norm

$$
\|\varphi\|=\iint_{R}|\varphi(z)| d x d y<\infty,
$$

(3) $\varphi$ is real along the sides of $R$ (i.e. $\varphi(z) d z^{2}$ real for tangential $d z$ ).

Because of (3) we also just speak of a real q.d. on $R$. Condition (2) implies that $\varphi$ can have poles of the first order at worst at the punctures $P_{\nu}$. We always exclude $\varphi \equiv 0$. Of course, if the border is empty, condition (3) is void.

Let $g: R \rightarrow R^{\prime}$ be a quasiconformal mapping of $R$ onto another such surface $R^{\prime}$. A mapping $g_{0}: R \rightarrow R^{\prime}$ which is homotopic to $g$ is called extremal in this class, if it has smallest maximal dilatation $K_{0}$. The homotopy is meant to keep each boundary component (as a whole) and each vertex fixed.

Teichmüller's theorem now says that for each $g$ there exists a unique extremal mapping $g_{0}$, and unless it is conformal it has the following structure. There is a pair of real quadratic differentials $\varphi$ on $R$ and $\psi$ on $R^{\prime}$ as described above, such that $g_{0}$ has locally and away from the critical points (zeros and poles) the representation

$$
g_{0}=\Psi^{-1} \circ F \circ \Phi
$$

with

$$
\begin{gathered}
\zeta=\Phi(z)=\int^{z} \sqrt{\varphi(z)} d z \\
\zeta^{\prime}=\Psi(w)=\int^{w} \sqrt{\psi(w)} d w
\end{gathered}
$$


and $F$ the horizontal stretching by $K_{0}$,

$$
F: \zeta=\xi+i \eta \rightarrow \zeta^{\prime}=K_{0} \xi+i \eta .
$$

The differentials $\varphi$ and $\psi$ are unique up to positive constant factors. They are called the Teichmüller differentials associated with $g$.

1.2. Elementary regions. One conceivable way to establish Teichmüller's theorem is to find the Teichmüller differentials associated with $g$ as solution of some extremum problem and then construct the mapping. In the general case there is no such method known. We will come back to this problem in a later paper. If, however, one can exclude that the solutions have recurrent trajectories, a deeper understanding of the so called quadratic differentials with closed trajectories leads to a direct construction. This is the case for plane domains of connectivity at most three (see [4], Section 15).

So let $G$ be a plane domain with at most three boundary components. By conformal mapping we can assume that they are analytic Jordan curves or points. At least one of the boundary components must be a curve, since otherwise, because of the finite norm, necessarily $\varphi \equiv 0$. The punctures are supposed to lie on the boundary. One example is the unit disk with finitely many distinguished boundary points (polygon), and possibly one or two interior point boundary components. In case of an annulus, we want to have at least one puncture on a boundary curve, to ensure uniqueness. We call these configurations "elementary regions". They give rise to what we call "the elementary cases in Teichmüller's mapping theorem".

1.3. Let now $G$ and $G^{\prime}$ be elementary regions, and let $g: G \rightarrow G^{\prime}$ be a qc mapping. We first set up a correspondence, induced by $g$, of the differentials associated with $G$ and those associated with $G^{\prime}$. This is possible in several ways, one way being by moduli of strips (as in [1], [2] and [3]). Here, we will however work with the heights of the strips. We then formulate an extremum problem involving the norm of quadratic differentials. A compactness argument and an elementary variational lemma leads to the Teichmüller differentials with which we can construct the Teichmüller mapping homotopic to $g$.

\section{Quadratic differentials with closed trajectories}

2.1. The trajectory structure. Let $\bar{G}$ be the closure of an elementary region and let $\varphi$ be a real quadratic differential on $\bar{G}$, i.e., enjoying properties (1), (2) and (3) of Section 1.1. Then, $\varphi$ can be reflected across the boundary curves of $G$ to become a symmetric differential $\tilde{\varphi}$ on the double $\tilde{G}$ of $\bar{G}$. The norm of $\tilde{\varphi}$ is twice the norm of $\varphi$. Conversely, if we start with a symmetric $\tilde{\varphi}$ on $\tilde{G}$, its restriction $\varphi$ to $\bar{G}$ is real. This 1-1-correspondence between the real differentials on $\bar{G}$ and the symmetric ones on $\tilde{G}$ will be used in the sequel.

Every non critical trajectory ray of a quadratic differential which belongs to a compact surface with punctures is either closed or recurrent. Let $\varphi$ be real 
on an elementary region $\bar{G}$. Because of the symmetry, its double $\tilde{\varphi}$ cannot have recurrent trajectory rays on $\tilde{G}$. Such a ray would have to leave $\bar{G}$ and come back into it, thus close up by reflection. Therefore $\tilde{\varphi}$ has closed trajectories. They partition $\tilde{G}$ into ring domains $\tilde{R}_{i}$. Each $\tilde{R}_{i}$ which contains points of $\partial G$ (in its interior) is bisected, either by two symmetric vertical intervals on $\partial G$ or by an entire boundary curve of $G$, which is then a closed trajectory of $\varphi$ and the middle line of $\tilde{R}_{i}$. In the first case the intersection $\tilde{R}_{i} \cap G$ is a quadrilateral with modulus $2 \tilde{M}_{i}$, in the second case it is a ring domain with modulus $\frac{1}{2} \tilde{M}_{i}$, where $\tilde{M}_{i}$ is the modulus of $\tilde{R}_{i}$. There is a slight complication: if there are punctures on $\partial G$ which are regular points of $\varphi$, there has to be subdivision of the corresponding domains along the horizontal trajectories through these points, because we always deal with the punctured surface $\dot{R}=R \backslash\left\{P_{\nu}\right\}$. With this in mind we can use the construction and theorems of [4], Chapter VI, rather than repeating the proofs for bordered surfaces.

2.2. The basic existence and uniqueness theorem. Let $\left\{\gamma_{i}\right\}$ be an admissible system of Jordan curves on a compact Riemann surface $R$ with finitely many punctures $P_{\nu}$ (for details see [4], Section 21). Then, to every system of non negative numbers $b_{i}$ there exists a quadratic differential $\varphi$ which is holomorphic and of finite norm on $R$ and has the following properties: its regular trajectories are closed, they lie in the free homotopy classes of the loops $\gamma_{i}$, and its cylinders $R_{i}$ have $\varphi$-height $b_{i}$. Of course, if for some $i, b_{i}=0$, there are no closed trajectories homotopic to $\gamma_{i}$, and we say that $R_{i}$ is degenerate.

The differential $\varphi$ is uniquely determined by these data (see [4], Theorem 21.1).

Let now $G$ be an elementary region as described above. An admissible system of curves consists of disjoint cross cuts and Jordan curves such that the double is an admissible system of Jordan curves of $\tilde{G}$. One point of caution: if a Jordan curve of the system is freely homotopic to a boundary curve $\Gamma$ of $G$ without punctures, then it is also freely homotopic, on $\tilde{G}$, to its mirror image. In that case we omit this mirror image in the system of Jordan curves on $\tilde{G}$. The associated annulus on $\tilde{G}$ will be bisected by $\Gamma$. We can now apply the above theorem to the double and then restrict the resulting quadratic differential to $G$. We get: for any system of positive numbers $b_{i}$ assigned to the given admissible curve system there is a unique real quadratic differential $\varphi$ with the prescribed topological determination and heights. (In order to get the correct heights one has to take into account the cutting and choose the heights for the double correspondingly.)

2.3. The surface of the squares of the heights. We will work here with the double of $G$, or more generally with a compact Riemann surface $R$ with punctures $P_{\nu}$ and an admissible system of Jordan curves $\gamma_{i}$ on $\dot{R}=R \backslash\left\{P_{\nu}\right\}$. The $\varphi$-heights of the cylinders are $b_{i}$, their circumferences are $a_{i}$, and their moduli $M_{i}=b_{i} / a_{i}$. For a fixed admissible system $\left\{\gamma_{i}\right\}$ the set of normed quadratic differentials $\varphi,\|\varphi\|=1$, has a remarkable structure. Namely, the vectors with 
the squares of the heights as components,

$$
x=\left(x_{1}, x_{2}, \ldots, x_{p}\right)=\left(b_{1}^{2}, \ldots, b_{p}^{2}\right)
$$

describe a concave surface $H$ in the positive quadrant of $p$-space, with normal vector

$$
y=\left(y_{1}, \ldots, y_{p}\right)=\left(\frac{1}{M_{1}}, \frac{1}{M_{2}}, \ldots, \frac{1}{M_{p}}\right) .
$$

The norm of $\varphi$ on $R$ is given by

$$
\|\varphi\|=\sum a_{i} b_{i}=\sum \frac{b_{i}^{2}}{M_{i}}=(x, y) .
$$

It is easy to see that in every non negative direction there is exactly one vector $x$ such that $(x, y)=1$. For, write the unit vector in the form $e=\left(e_{1}, \ldots, e_{p}\right)$, and denote the quadratic differential with heights $\sqrt{e_{i}}$ by $\varphi_{0}$. Then $\varphi=\lambda \varphi_{0}$, $\lambda=\left\|\varphi_{0}\right\|^{-1}$ has the desired property. Its heights are

$$
b_{i}=\int_{\beta_{i}}|\varphi(z)|^{1 / 2}|d z|=\sqrt{\lambda} \sqrt{e_{i}}
$$

with $\beta_{i}$ a vertical cross cut of $R_{i}$. Thus $b_{i}^{2}=\lambda e_{i}$, and $\varphi$ is clearly the only solution.

The surface $H$ is concave, continuously differentiable at its interior points $x_{i}>0$ for all $i$, and $y$ is the normal at the point $x$ (see [4], Section 21.6).

The result can easily be translated to the case of elementary regions, with corresponding moduli and heights, but we rather work with the double.

\section{The "mapping by heights" and a norm inequality}

3.1. The "mapping by heights". The existence and uniqueness theorem of Section 2.2 can be used to establish a correspondence between the quadratic differentials with closed trajectories of two surfaces. Let $R$ and $R^{\prime}$ be compact surfaces with punctures $P_{\nu}, P_{\nu}^{\prime}$ respectively and let $g: R \rightarrow R^{\prime}$ be a homeomorphism, $g\left(P_{\nu}\right)=P_{\nu \text {. }}^{\prime}$ for all indices $\nu$. Let $\varphi$ be a holomorphic quadratic differential of finite norm on $\dot{R}=R \backslash\left\{P_{\nu}\right\}$, with closed trajectories. The trajectory structure of $\varphi$ partitions $\dot{R}$ into ring domains $R_{i}$, with $\varphi$-heights $b_{i}$, say. Pick a closed trajectory $\gamma_{i}$ out of every $R_{i}$. The system of Jordan curves $\gamma_{i}^{\prime}=g\left(\gamma_{i}\right)$ is admissible on $\dot{R}^{\prime}=R^{\prime} \backslash\left\{P_{\nu}^{\prime}\right\}$. Denote by $\psi$ the quadratic differential with closed trajectories on $\dot{R}^{\prime}$ which is associated with the curve system $\left\{\gamma_{i}^{\prime}\right\}$ and such that the cylinders $R_{i}^{\prime}$ have $\psi$-heights $b_{i}^{\prime}=b_{i}$. The correspondence is the "mapping by heights" $g_{H}$ ( $H$ for "height") induced by the homeomorphism $g$. It is not hard to see that it is a special case of the general mapping by heights of [5] and [6] (see 
[4], Section 25.5). It only depends on the homotopy class of $g$ and is actually a homeomorphism of the set of quadratic differentials with closed trajectories on $\dot{R}$ onto the corresponding set of $\dot{R}^{\prime}$.

Let now $\bar{G}, \bar{G}^{\prime}$ be the closures of two elementary domains and let $g: \bar{G} \rightarrow \bar{G}^{\prime}$ be a homeomorphism. We can continue $g$ to $\tilde{G}$ by reflection. Let $\varphi$ be a real quadratic differential of $\bar{G}$. Continuing $\varphi$ to $\tilde{\varphi}$ by reflection, passing to the image by heights $\tilde{\psi}$ and then restricting to $\psi$ on $\bar{G}^{\prime}$ we find the mapping by heights $g_{H}$ of the real differentials on $\bar{G}$ to those on $\bar{G}^{\prime}$. They both have the same heights on corresponding strips (quadrilaterals and annuli).

3.2. The norm inequality. Assume now that $g: \bar{G} \rightarrow \bar{G}^{\prime}$ is a quasiconformal mapping with maximal dilatation $K$. Then the following inequality for the norms of the quadratic differentials holds.

Theorem 3.2. Let $\psi=g_{H}(\varphi)$. We have

$$
\|\psi\| \leq K\|\varphi\|
$$

with equality if and only if $g$ is a Teichmüller mapping with dilatation $K$ and with Teichmüller differentials $\varphi$ and $\psi$ respectively.

Proof. We pass to the doubles, thereby multiplying both norms by two. We therefore work with two compact surfaces $R$ and $R^{\prime}$ with punctures. Let $\varphi$ be a quadratic differential with closed trajectories on $\dot{R}=R \backslash\left\{P_{\nu}\right\}$, and let $b_{i}, M_{i}$ be the heights and moduli respectively of the induced ring domains $R_{i}$. The $R_{i}$ are taken, by $g$, into ring domains $\tilde{R}_{i}$ on $R^{\prime}$, with moduli $\tilde{M}_{i}$. Then,

$$
\frac{1}{K} M_{i} \leq \tilde{M}_{i} \leq K M_{i}, \quad i=1, \ldots, p .
$$

From the left hand inequality we get, after multiplication with $b_{i}^{2}$,

$$
\frac{b_{i}^{2}}{\tilde{M}_{i}} \leq K \frac{b_{i}^{2}}{M_{i}}
$$

and by summation,

$$
\sum_{i} \frac{b_{i}^{2}}{\tilde{M}_{i}} \leq K \sum_{i} \frac{b_{i}^{2}}{M_{i}}
$$

The partitioning $\left\{R_{i}^{\prime}\right\}$ induced by $\psi$ is minimal (see, [4], Theorem 20.5). Therefore the moduli $M_{i}^{\prime}$ of the $R_{i}^{\prime}$ satisfy

$$
\sum_{i} \frac{b_{i}^{2}}{M_{i}^{\prime}} \leq \sum_{i} \frac{b_{i}^{2}}{\tilde{M}_{i}} \leq K \sum_{i} \frac{b_{i}^{2}}{M_{i}}
$$


With the equalities

$$
\|\psi\|=\sum_{i} \frac{b_{i}^{2}}{M_{i}^{\prime}}, \quad\|\varphi\|=\sum_{i} \frac{b_{i}^{2}}{M_{i}}
$$

this proves inequality (0).

Assume that there is a q.d. $\varphi$ with closed trajectories on $\dot{R}$ such that equality holds in (0). Then, we must have equality in the left hand side of (4), and hence because of the uniqueness of the extremal partitioning, $\tilde{R}_{i}=R_{i}^{\prime}, i=1, \ldots, p$. Furthermore, equality must hold in the left hand side of (1), which gives

$$
\tilde{M}_{i}=\frac{1}{K} M_{i}, \quad i=1, \ldots, p .
$$

Since the mapping $g: R_{i} \rightarrow \tilde{R}_{i}$ is $K$-qc, this can only happen if $g$ is an extremal mapping of $R_{i}$ onto $\tilde{R}_{i}=R_{i}^{\prime}$. Because $R_{i}$ and $R_{i}^{\prime}$ have the same heights in terms of $\varphi$ and $\psi$ respectively, this is a stretching along the trajectories of $\varphi$.

The same must then be true at the points of the critical graph. For, let $z$ be a point on a critical trajectory of $\varphi$. Then, $w=g(z)$ must lie on the critical graph of $\psi$, since it cannot lie in a ring domain $R_{i}^{\prime}=g\left(R_{i}\right)$. The integral $\Phi$ maps a neighborhood $U$ of $z$ conformally into the $\zeta$-plane, and $g$ corresponds to a horizontal stretching by $K$ in both the upper and the lower half neighborhoods. We conclude that the same must hold on the critical trajectory interval in $U$. In particular, $w$ is a regular point of a critical trajectory of $\psi$. The argument can be adapted to critical points and one finds that zeroes of $\varphi$ are mapped onto zeroes of $\psi$ of the same order. Of course we have always made use of the fact that we have a given qc mapping $g$.

It is known that a Teichmüller mapping $g$ associated with a q.d. of finite norm is uniquely extremal, and moreover that $\varphi$ and hence $\psi$ are uniquely determined up to positive constant factors. (Extremality is actually evident here, since we can start with an extremal $g$.)

Let us now start with a real differential $\varphi$ on an elementary region $\bar{G}$. Then, its continuation $\tilde{\varphi}$ to the double $\tilde{G}$ has closed trajectories, and so does $\tilde{\psi}$. We can therefore apply the above argument. In the case of equality $\|\psi\|=K\|\psi\|$ in $G$, we have equality $\|\tilde{\psi}\|=K\|\tilde{\varphi}\|$ in $\tilde{G}$. Therefore $g: \tilde{G} \rightarrow \tilde{G}^{\prime}$ is a Teichmüller mapping, and because of the symmetry, it can be restricted to $G$.

\section{The extremum problem}

4.1. There is no a priori evidence that there exists a qc map $g: G \rightarrow G^{\prime}$ for which equality holds in (0). Therefore we will work with the supremum

$$
L=\sup _{\{\varphi\}}\left\{\frac{\|\psi\|}{\|\varphi\|}, \frac{\|\varphi\|}{\|\psi\|}\right\}, \quad \psi=g_{H}(\varphi) .
$$


The space $\{\varphi\}$ is a finite dimensional vector space over the real numbers, and the mapping by heights $g_{H}$ is continuous (which follows easily from [4], Section 21.6). Therefore there exists at least one extremal pair of differentials. By the definition $L \geq 1$, and we may assume that

$$
L=\frac{\|\psi\|}{\|\varphi\|}
$$

4.2. The variational lemma. Let $\varphi$ be an extremal quadratic differential on $G, \psi=g_{H}(\varphi)$. Again we pass to the double by reflection. We are therefore back to compact surfaces with punctures, and we formulate the auxiliary result for this situation.

Lemma 4.2. Let $R$ and $R^{\prime}$ be compact Riemann surfaces with punctures, and let $g: R \rightarrow R^{\prime}$ be a homeomorphism. Let $\varphi$ have closed trajectories and finite norm on $\dot{R}=R \backslash\left\{P_{\nu}\right\}$ and assume that $L=\|\psi\| /\|\varphi\|$ is maximal. Then, there is a positive number $\lambda$ such that the moduli (see Section 3.2) satisfy

$$
\frac{1}{M_{i}^{\prime}}=\lambda \frac{1}{M_{i}}, \quad i=1, \ldots, p .
$$

Proof. We pick a closed trajectory $\gamma_{i}$ out of every ring domain $R_{i}$ of $\varphi$. This is an admissible system of Jordan curves on $\dot{R}$, while the curves $\gamma_{i}^{\prime}=g\left(\gamma_{i}\right)$ form an admissible system on $\dot{R}^{\prime}=R^{\prime} \backslash\left\{P_{\nu}^{\prime}\right\}$. We look at the normalized quadratic differentials $\varphi,\|\varphi\|=1$, with closed trajectories of homotopy type $\gamma_{i}$. Let $H$ be the surface of the squares of heights (Section 2.3). The differentials $\psi=g_{H}(\varphi)$ are normalized by the requirement that $\|\psi\|=L$. Let $H^{\prime}$ be the corresponding surface for $\dot{R}^{\prime}$. The two surfaces have the point $x$ in common, whereas in all other directions $H$ lies to the left of $H^{\prime}$ (i.e. closer to the origin). Therefore the two normal vectors $y$ and $y^{\prime}$ at $x$ must be parallel, which is the relation claimed in the lemma. (Of course, if $L=1$, both surfaces $H$ and $H^{\prime}$ coincide.)

We will use the lemma for the differentials on $G$ and $G^{\prime}$ respectively. But the restrictions in the different ring domains are the same on both sides. Therefore it is also true.

At the common point $x$ of the two surfaces the two differentials $\varphi$ and $\psi$ have the same heights $b_{i}=b_{i}^{\prime}$. Therefore $a_{i}^{\prime}=\lambda a_{i}$. From

$$
\|\psi\|=\sum a_{i}^{\prime} b_{i}=\lambda \sum a_{i} b_{i}=\lambda\|\varphi\|=L\|\varphi\|
$$

we get $\lambda=L$.

(If there is only one ring domain, the lemma is trivial.)

4.3. The basic fact we need for the construction of the Teichmüller mapping which is homotopic to $g$ is that for the extremal pair $g_{H}$ not only takes the horizontal trajectories of $\varphi$ into those of $\psi$, but also the vertical ones into the vertical ones (see [6]). 
Lemma 4.3. Let $\left\|g_{H}(\varphi)\right\|=L\|\varphi\|$, and let $L \geq 1$ be maximal. Then

$$
g_{H}(-\varphi)=\frac{1}{L^{2}}(-\psi) \text {. }
$$

Proof. We start with the differential $-\psi$ on the right hand side. It is real on $\bar{G}^{\prime}$ and subdivides $G^{\prime}$ into strips $S_{j}^{\prime}$ (quadrilaterals and ring domains) with lengths and heights $c_{j}^{\prime}$ and $d_{j}^{\prime}$ respectively. Notice that the heights are along the trajectories, the lengths along the vertical trajectories of $\psi$.

Let $\tilde{\varphi}=g_{H}^{-1}(-\psi)$, and let $\tilde{S}_{j}$ be the strips of $\tilde{\varphi}$, corresponding to the $S_{j}^{\prime}$ with heights $\tilde{d}_{j}=d_{j}^{\prime}$ and lengths $\tilde{c}_{j}$. If the vertical trajectory $\beta_{j}^{\prime}$ in $S_{j}^{\prime}$ cuts the horizontal trajectory $\alpha_{i}^{\prime}$ in $R_{i}^{\prime}$, then the trajectories $\tilde{\beta}_{j}$ of $\tilde{\varphi}$ in $\tilde{S}_{j}$ cut the horizontal trajectory $\alpha_{i}$ of $\varphi$ in $R_{i}$. This is so because the number of crossings in $G^{\prime}$ is in fact the geometric intersection number which is invariant under a homeomorphism. (For simply connected domains, the number of crossings in $G^{\prime}$ is zero or one; it can be at most two in the other cases.)

We now compute the norm of $\tilde{\varphi}$, using the strips $R_{i}$ of $\varphi$ as parameter domains and representing $\tilde{\varphi}$ in terms of the parameter $\Phi$, which we call $z$ again.

Let $\alpha_{i}$ be a trajectory of $\varphi$ in $R_{i}$, and let $d \tilde{w}=\sqrt{\tilde{\varphi}(z)} d z$. Then,

$$
\int_{\alpha_{i}}|d \tilde{w}|=\int_{\alpha_{i}}|\tilde{\varphi}(z)|^{1 / 2} d x \geq \sum \tilde{d}_{j}=\sum d_{j}^{\prime}=a_{j}^{\prime}=L a_{i}
$$

where the sum is taken over all strips $\tilde{S}_{j}$ traversed by $\alpha_{i}$. Integrating over the strips of $\varphi$ and summing we find successively

$$
\begin{gathered}
\iint_{R_{i}}|\tilde{\varphi}(z)|^{1 / 2} d x d y \geq L a_{i} b_{i} \\
\iint_{G}|\tilde{\varphi}(z)|^{1 / 2} d x d y \equiv \sum_{i} \iint_{R_{i}}|\tilde{\varphi}(z)|^{1 / 2} d x d y \geq L \sum a_{i} b_{i}=L\|\varphi\| .
\end{gathered}
$$

By means of the Schwarz inequality we get

$$
L^{2}\|\varphi\|^{2} \leq\|\varphi\| \cdot \iint_{G}|\tilde{\varphi}(z)| d x d y=\|\varphi\| \cdot\|\tilde{\varphi}\|,
$$

and hence

$$
\|\tilde{\varphi}\| \geq L^{2}\|\varphi\|=L\|\psi\| .
$$

Evidently, because of the extremality, the equality sign must hold. Therefore, $|\tilde{\varphi}(z)|=$ const. From (1) we conclude that the strips $\tilde{S}_{j}$ must be traversed vertically, i.e. the vertical trajectories of $\tilde{\varphi}$ are the horizontal trajectories of $\varphi$, which means that $\tilde{\varphi}=t(-\varphi)$ with a positive constant $t$.

In order to match the stretching of the horizontal trajectories of $\varphi$ by $L$, we replace the mapping by heights for $-\varphi$ by the stretching of the heights by $L$. We thus have a mapping of the horizontal and vertical strips of $\varphi$ onto the horizontal and vertical strips of $\psi$. On this basis we can construct the Teichmüller mapping which is homotopic to $g$. 


\section{Construction of the Teichmüller mapping}

5.1. In [6] the following characterization of Teichmüller differentials was given.

Let $g: R \rightarrow R^{\prime}$ be a homeomorphism of compact surfaces with punctures. We denote by $Q_{0}$ and $Q_{0}^{\prime}$ the subsets of normed quadratic differentials of $R$ and $R^{\prime}$ respectively. The normed mapping by heights $g_{\#}$ maps $Q_{0}$ onto $Q_{0}^{\prime}$ in such a way that corresponding heights are proportional (not necessarily the same). Then, $\varphi$ and $\psi=g_{\#}(\varphi)$ are the Teichmüller differentials associated with $g$ if and only if $g_{\#}(-\varphi)=-g_{\#}(\varphi)$. The definition of "height" is more general then we use it here, but it is easy to see, applying geometric intersection numbers, that ours is in fact a special case. If we work with the double rather than the elementary region itself, we have exactly the situation of the theorem. By the symmetry of the data and the uniqueness of the differentials and the mapping, we can restrict the result to $G$. This proves the existence of an extremal Teichmüller mapping homotopic to $g$.

5.2. In the case of a simply connected region with distinguished points on the boundary (polygon) two mappings $f$ and $g$ are homotopic if and only if they agree on the vertices. We can then construct the Teichmüller mapping $f$ homotopic to $g$ without referring to the above mentioned theorem.

Let $g: G \rightarrow G^{\prime}$ be a qc mapping of two polygons, and let $\varphi$ be the solution of the extremum problem 4.1 with respect to $g$. As shown in 4.3 , the decompositions of $G$ into horizontal strips $R_{i}$ and into vertical strips $S_{j}$ induced by $\varphi$ correspond to the decompositions $\left\{R_{i}^{\prime}\right\},\left\{S_{j}^{\prime}\right\}$ of $G^{\prime}$ with respect to $\psi=g_{H}(\varphi)$. The heights of the horizontal strips $R_{i}$ and $R_{i}^{\prime}$ are the same, whereas the lengths are multiplied by $L, a_{i}^{\prime}=L a_{i}$. On the other hand, the heights of the vertical strips $S_{j}^{\prime}$ of $\psi$ are $L$ times the heights of the vertical strips $S_{j}$ of $\varphi$, which again is a streching by $L$ along the trajectories.

It is easy to figure out that these maps fit together to a Teichmüller mapping $f$, with dilatation $L$, associated with the differentials $\varphi$ and $\psi=g_{H}(\varphi)$. To every regular horizontal trajectory $\alpha$ of $\varphi$ corresponds a unique horizontal trajectory $\alpha^{\prime}$ of $\psi$ by the requirement that $\alpha^{\prime}$ subdivides the horizontal strip $R_{i}^{\prime}$ in the same proportion as $\alpha$ subdivides $R_{i}$. Likewise, to every regular vertical trajectory $\beta$ of $\varphi$ corresponds a vertical trajectory $\beta^{\prime}$ of $\psi$. Assume now that $\alpha$ and $\beta$ intersect at a point $z \in G$. Then, they join sides of $G$ which are separated by vertices. By construction, the same is true for $\alpha^{\prime}$ and $\beta^{\prime}$. It follows that $\alpha$ and $\beta$ cut in a point $z$ if and only if $\alpha^{\prime}$ and $\beta^{\prime}$ have an intersection $w$.

We now define $f(z)=w$. This is a bijection of the intersections of regular horizontal and vertical trajectories. It is evident that every $\varphi$-rectangle $R_{i} \cap S_{j}$ is thus horizontally stretched by $L$ onto the $\psi$-rectangle $R_{i}^{\prime} \cap S_{j}^{\prime}$. We therefore have a piecewise Teichmüller mapping.

Let now $\alpha$ be a regular horizontal trajectory. It is contained in a horizontal strip $R_{i}$ and thus in rectangles $R_{i} \cap S_{j}$ except for its finitely many intersections with critical vertical trajectories. Evidently, the intervals $\alpha \cap S_{j}$ correspond to the 
intervals $\alpha^{\prime} \cap S_{j}^{\prime}$, and thus the intersections of $\alpha$ with critical vertical trajectories of $\varphi$ to the intersections of $\alpha^{\prime}$ with critical vertical intersections of $\psi$. We conclude that the map $f$ stretches each non critical trajectory $\alpha$ of $\varphi$ to a well defined trajectory $\alpha^{\prime}$ of $\psi$ by the factor $L$.

Similarly, every vertical trajectory $\beta$ of $\varphi$ is mapped onto a well defined vertical trajectory $\beta^{\prime}$ of $\psi$, preserving lenghts. What remains are the finitely many intersections of a critical horizontal and a critical vertical trajectory, and the finitely many critical points. But the map $f$ can evidently be extended to these too, and we thus have a Teichmüller map $f$ with dilatation $L$, associated with the differentials $\varphi$ on $G$ and $\psi$ on $G^{\prime}$.

The argument applies to the sides of $G$ as well, which are composed of horizontal and vertical intervals of $\varphi$. They are therefore mapped by $f$ onto the corresponding sides of $G^{\prime}$. This shows that $f$ and $g$ are identical at the vertices and are thus homotopic.

Remark. From the uniqueness of the Teichmüller mapping in case $L>1$, we conclude that the extremum problem 4.1 has only one solution.

\section{References}

[1] Strebel, K.: Über quadratische Differentiale mit geschlossenen Trajektorien und extremale quasikonforme Abbildungen. - Festband zum 70. Geburtstag von Rolf Nevanlinna, Springer-Verlag, Berlin-New York-Heidelberg, 1965/66, 105-127.

[2] Strebel, K.: Bemerkungen über quadratische Differentiale mit geschlossenen Trajektorien. - Ann. Acad. Sci. Fenn. Ser. A I Math. 405, 1967, 1-12.

[3] Strebel, K.: On quadratic differentials and extremal quasiconformal mappings. - Lecture Notes, University of Minnesota, Minneapolis, 1967, 1-112.

[4] Strebel, K.: Quadratic differentials. - Ergebnisse der Mathematik und ihrer Grenzgebiete, 3. Folge Bd 5. Springer-Verlag, Berlin-New York-Heidelberg, 1984, 1-184.

[5] Marden, A., and K. Strebel: The heights theorem for quadratic differentials on Riemann surfaces. - Acta Math. 153, 1984, 153-211.

[6] Marden, A., and K. Strebel: A characterization of Teichmüller differentials and the unique axis theorem for Teichmüller space. - Preprint 1989.

University of Zurich

Department of Mathematics

CH-8001 Zürich

Switzerland

Received 8 March 1990 